\title{
Analyzing Vibration Suppression of Nuclear Power Crane with Model Coupling Mechanism and Structure
}

\author{
Yixiao Qin ${ }^{1}$, Qingqing Yang ${ }^{1}$, , Yanqing $\mathbf{L i}^{1}$, Cuiyun $\mathbf{G u}^{2}$ \\ ${ }^{1}$ Mechanical Engineering Institution, Taiyuan University of Science and Technology, Taiyuan, Shanxi, China \\ ${ }^{2}$ Taiyuan Heavy Industry Limited by Share Ltd, Taiyuan, Shanxi, China
}

Email address:

744257441@qq.com (Qingqing Yang)

${ }^{*}$ Corresponding author

\section{To cite this article:}

Yixiao Qin, Qingqing Yang, Yanqing Li, Cuiyun Gu. Analyzing Vibration Suppression of Nuclear Power Crane with Model Coupling Mechanism and Structure. International Journal of Mechanical Engineering and Applications. Vol. 4, No. 4, 2016, pp. $136-142$. doi: 10.11648/j.ijmea.20160404.11

Received: May 24, 2016; Accepted: June 5, 2016; Published: June 18, 2016

\begin{abstract}
The nuclear power crane possesses strict safety requirements. Based on the special structure of its hoisting mechanism, it has two sets of independent lifting ropes to drive a hook synchronously and two pressure buffer devices on both ends of winding system's balancing lever, in order to play protective roles and increase its working reliability. The flexible multi-body dynamics model which coupled mechanism and structure of the nuclear power crane is constructed when broken accident of rope occurs in the lifting process from the ground or smooth lifting. The importance of a buffer damping device installed in crane hoist mechanism has been proved through the vibration simulation about wire rope at the failure state. And the vibration of mechanism and the main girder structure is remitted and controlled at the time of failure in operation. The most important advantage is that has improved the safety of nuclear power crane.
\end{abstract}

Keywords: Nuclear Power Crane, Multi-body Dynamics, Coupling Mechanism and Structure, Rope Breaking Fault, Vibration Suppression

\section{Introduction}

Nuclear energy, as a clean energy with advanced technology and high supply capacity, has very important implications in meeting increasing energy demands of the socially rapid development and realizing power structure optimization, even economically sustainable development. Nuclear power crane is one of the important equipment about nuclear power plant construction and operation, which is primarily composed of bridge structure, the central arch, rotating mechanism, running trolley, installed trolley, anti-vibration device and electrical control system etc. Compared with ordinary crane, nuclear power crane has more strict quality requirements and higher design standards. Because of particularity of place to work, special crane for nuclear power station must be ensured safety, reliable operation and accurate positioning. In addition, the importance of crane operation safety is more prominent as a result of frequent crane accidents. Therefore, it is significant to study the dynamic features at the fault state of nuclear power crane. Although the research results on dynamics of nuclear power crane at the fault state are few at present, a lot of work on crane dynamics have been already done. Severe vibration will make beam crack from the measured amplitudes at two points of the structure vibrating at one of its natural modes, the respective vibration frequency and an analytical solution of the dynamic response, the crack location can be found and depth can be estimated with satisfactory accuracy, which is applicable to structures and structural analysis is available [1]. A time-stepping model of a transversely vibrating was formulated by using a time-stepping approach [2]. Relaxation damping was a phenomenon described recently for contact of two purely elastic bodies with infinite coefficient of friction. A model of a breathing crack with relaxation damping was established [3]. The continuous cracked beam vibration theory and a lumped cracked beam vibration analysis were developed [4]. An analysis of the effect of two open cracks upon the 
frequencies of the natural flexural vibrations in a cantilever beam was presented as a new method [5]. The mechanics for shell finite element for smart piezoelectric composite plate/shell structures were discussed and it is applied to the study of active vibration control [6]. A mechatronic approach integrating both passive and active controllers was presented in this study to deal with unwanted noise and vibration produced in an automobile wiper system operation [7]. After introducing the concept of modified positive position feedback, the stability of the new method for locating gain limits was investigated, the proposed method was significantly more effective in controlling steady-state response and slightly advantageous for transient dynamics control [8]. One great advantage of positive position feedback control was the frequency response of the controller rolls off quickly [9]. The synchronized switch damping technique has been demonstrated as an efficient means of suppressing structure vibrations, a novel switch damping technique based on an energy transfer scheme that transmits energy from an energy-source structure to a target structure in order to damp the latter was presented [10]. A hybrid control scheme to vibration reduction of flexible spacecraft has been proposed by using a variable structure technique for attitude control and piezoelectric materials for active vibration suppression [11]. A controller for uncertain structures that are minimum phase and potentially subject to unknown and unmeasured disturbances, was designed [12]. A method for the dynamic analysis of elastic beams subjected to dynamic loads induced by the arbitrary movement of a spring mass damper system was presented [13]. The theoretical analysis of nonlinear micro beams, the static bending, postbuckling and free vibration were studied [14]. An alternative method about artificial neural networks was introduced which used to obtain numerical solutions of mathematical models of dynamic systems, the mathematical models of the control of longitudinal vibrations of rods and lateral vibrations of beams have been considered. Finally, the responses of the controlled and non-controlled systems have been researched [15]. A neutral-type time-delay model that effectively describes the oscillatory behavior in the drilling system was researched. The attractive ellipsoid method allows us to develop an efficient strategy for the stabilizing controllers synthesis guaranteeing the elimination of drilling oscillations [16]. Simulation was carried out by using SIMULINK, the time domain and frequency domain in response to main beams have been analyzed, the same test also has been done for weight [17]. The nuclear power crane is different from common cranes, buffer device is installed in its hoisting mechanism. And these buffer cylinder devices can effectively improve lifting safety and stability of nuclear crane, slow down the vibration amplitude of the structure and mechanism coupling system and improve the operation safety at the fault state. For the nuclear power crane hoisting mechanism of the special structure, the vibration of coupling mechanism and structure at the time of failure in operation is researched. This study will improve the safety of the operation of nuclear power crane.

\section{Flexible Multi-body Dynamic Model of Coupling Mechanism and Structure}

\subsection{The Particularities of Lifting Mechanism of Nuclear Power Crane}

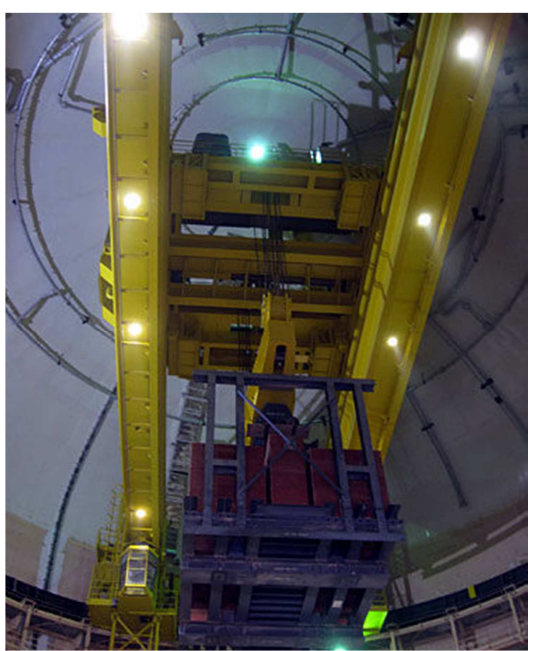

Fig. 1. Nuclear crane with circular orbit and diameter for 35 meters.

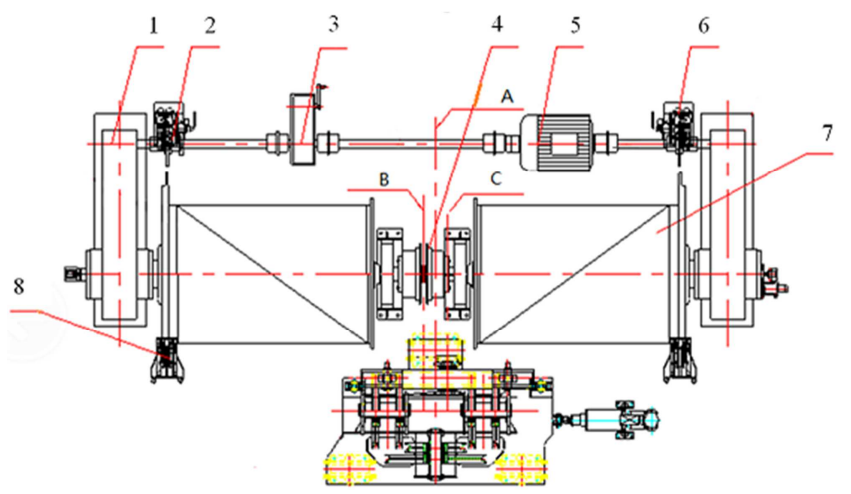

Fig. 2. Drive lifting mechanism of double velocity reducer.

The marks in the figure are: 1 . Retarder; 2 . Working brake; 3 . Manual retarder; 4. Couplings; 5 . Motors; 6 . Mergency brake; 7. Reel; 8 . Safety brake;

A. The center line of the fixed pulley and hook; B. The center line of ree; C. The center line of balance lever

A crane with circular orbit for nuclear power reactors in the workshop is shown in Fig. 1, which consists of dual-beam bridge structure and heavy running trolley on them.

There is lifting mechanism with high-reliability on the lower part of the trolley, the mechanism consists of a front end wire winding system and a rear end power drive mechanism, as shown in Fig. 2.

The dynamic model at normal working state is shown in Fig. 1 , balanced beams are set on the two rope tails of single winding system, which are driven independently and hoisted together on the trolley. Damping cylinder is used to prop on both ends of trolley frame and the balanced beam, due to the relaxations of the rope on one end of the balanced beam, the other side of the tension rope acts as cushioning when making its buffer device be under pressure. Buffer tank does not work when two sets of power plants work normally. 


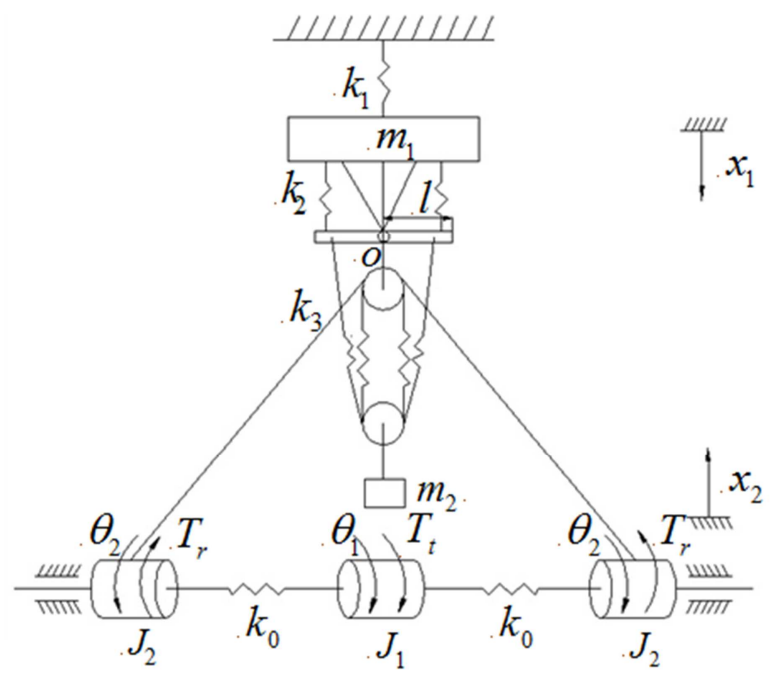

Fig. 3. Dynamic model of the system in normal operation.

In Fig. 3, the symbols of every physical quantities are: moment of inertia of motor rotor and coupling $J_{1}$, the rotational inertia of the brake wheel $J_{2}$, conversion quality of structure in suspension point $m_{1}$, quality of banging device and effective load $m_{2}$, the stiffness coefficient of drive shafts $k_{0}$, damping of drive shafts $c_{0}$, stiffness coefficient of structure in suspension $k_{1}$, damping of structure in suspension point $c_{1}$, the stiffness coefficient of the pulley block $k_{2}$, damping of pulley block $c_{2}$, stiffness coefficient of connecting balanced rod $k_{3}$, damping of connecting balanced $\operatorname{rod} c_{3}$, starting torque of motor $T_{t}$, the mechanism static resistance torque $T_{r}$, radius of drum $r$, ratio of pulley block $n$, velocity ratio of mechanism $i$. Among them, $J_{1}$ consists of the rotary inertia of the rotor and half of turning inertia of coupling, $m_{1}$ consists of mass of the trolley and half of main beam quality.

A comparative study is carried out aimed at dynamic characteristics of crane lifting normally operation and rope being broken still to work continuously. Crane is simplified into elastic vibration system of limited mass and multi-degree of freedom, at this point, which is an elastic system with multiple degrees of freedom and more quality. Its dynamic characteristics are studied through simplified into elastic vibration system of coupling lifting mechanism and bridge structure. When crane lifting mechanism works normally, the maximum dynamic response occurs in these two conditions: the cargo is lifted from the ground and drops braking at full velocity. The dynamic response problem is studied, the moment that cargo was hanging.

\subsection{Multi-body Dynamic Model as Running Normally}

The mechanical model of the system in normal operation is shown in Fig. 3. When the system is running normally, buffer tank does not work. At this time, the dynamic equations of the system are

$$
M \ddot{x}+C \dot{x}+K x=F
$$

Here, vector of degree freedom in system $x$, external load vector $F$, mass matrix $M$, damping matrix $C$, and stiffness matrix $K$ are respectively

$$
\begin{gathered}
x=\left[\begin{array}{llll}
\theta_{1} & \theta_{2} & x_{1} & x_{2}
\end{array}\right]^{\mathrm{T}}, \\
F=\left[\begin{array}{llll}
T_{t} & T_{r} & 0 & 0
\end{array}\right]^{\mathrm{T}}, \\
M=\left[\begin{array}{cccc}
J_{1} & 0 & 0 & 0 \\
0 & J_{2} & 0 & 0 \\
0 & 0 & m_{1} & 0 \\
0 & 0 & 0 & m_{2}
\end{array}\right],
\end{gathered}
$$

$$
C=\left[\begin{array}{cccc}
2 c_{0} & -2 c_{0} & 0 & 0 \\
-c_{0} & c_{0}+\left(\frac{r}{i n}\right)^{2} c_{2} & -\frac{r}{i n} c_{2} & -\frac{r}{i n} c_{2} \\
0 & -2 \frac{r}{i n} c_{2} & c_{1}+c_{2} & c_{2} \\
0 & -2 \frac{r}{i n} c_{2} & c_{2} & c_{2}
\end{array}\right],
$$

$$
K=\left[\begin{array}{cccc}
2 k_{0} & -2 k_{0} & 0 & 0 \\
-k_{0} & k_{0}+\left(\frac{r}{i n}\right)^{2} k_{2} & \frac{r}{i n} k_{2} & \frac{r}{i n} k_{2} \\
0 & -2 \frac{r}{i n} k_{2} & k_{1}+k_{2} & k_{2} \\
0 & -2 \frac{r}{i n} k_{2} & k_{2} & k_{2}
\end{array}\right] .
$$

\subsection{Multi-body Dynamic Model with Sudden Failure and Without Buffer Device}

Space cross winding scheme is used in system of wire rope winding of lifting mechanism. As shown in Fig. 2, when a set of power plant is broken, that is, a wire rope is broken, as shown in Fig. 4, balanced beam deflects and vibrates around the $O$ point. At this moment, the damping oil cylinder plays a buffer role, which reduces dynamic shock of instantaneous failure, in order to avoid fatal damage to the system, at the same time, also ensures the safety and stability of the lifting mechanism and the whole machine.

If not provided with buffer and damping device in the system, while a wire rope is suddenly broken, the vibration equation is

$$
M_{1} \ddot{x}+C_{1} \dot{x}+K_{1} x=F_{1}
$$


Where, degree of freedom vector in system $x$ which is unchanged, mass matrix $M$, external load vector $F$, damping matrix $C_{1}$, stiffness matrix $K_{1}$ become respectively

$$
\begin{gathered}
F_{1}=\left[\begin{array}{llll}
T_{t} & 2 T_{r} & 0 & 0
\end{array}\right]^{\mathrm{T}}, \\
M_{1}=\left[\begin{array}{cccc}
J_{1} & 0 & 0 & 0 \\
0 & 2 J_{2} & 0 & 0 \\
0 & 0 & m_{1} & 0 \\
0 & 0 & 0 & m_{2}
\end{array}\right], \\
C_{1}=\left[\begin{array}{cccc}
c_{0} & -c_{0} & 0 & 0 \\
-c_{0} & c_{0}+\left(\frac{r}{i n}\right)^{2} & -\frac{r}{i n} c_{2} & -\frac{r}{i n} c_{2} \\
0 & -\frac{r}{i n} c_{2} & c_{1}+c_{2} & c_{2} \\
K_{1}= & -\frac{r}{i n} c_{2} & c_{2} & c_{2}
\end{array}\right], \\
0
\end{gathered}
$$

\subsection{Multi-body Dynamic Model with a Buffer Device When Either of Two Wire Ropes Is Broken}

By the rope winding method of crane lifting mechanism, the load distribution on two wire ropes can be balanced, and there are two wire ropes, if one is broken, the other can keep load, so the balance of the spreader is maintained. Meanwhile this winding method of wire rope also avoids hook's horizontal movement during the lifting process. Buffer cylinders are installed at both ends of the balance lever device, its function is that another rope can bear all the load in the buffer mode after one of the two wire ropes is broken. Choosing rationally buffer cylinder can reduce load impact factor caused by sudden rupture of a wire rope and reducing short-time maximum impact load next wire rope bears under the condition of the accident. A dynamic model is established when system is in fault. To verify that vibration caused by lifting mechanism buffer cylinder has a certain attenuation, and accordingly the advantages of the system and the fidelity of the model can also be verified.

As shown in Fig. 4. and Fig. 5, there are a system failure when one rope is suddenly broken, balancing rod deflects around the rotating shaft $O$, adding one degree of freedom

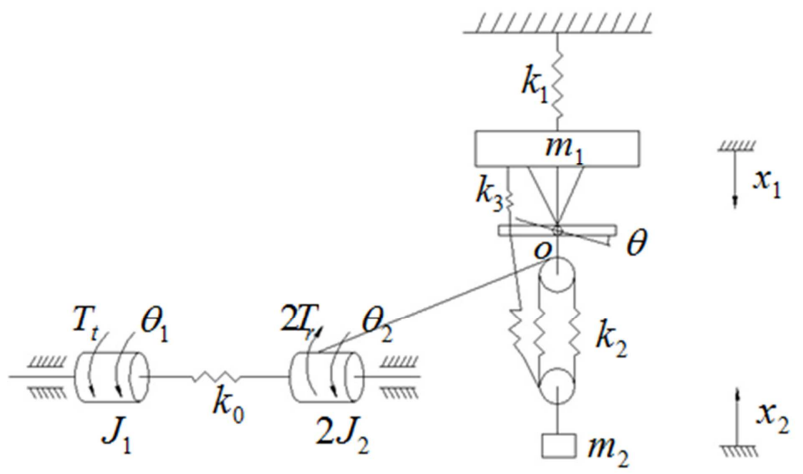

Fig. 4. Dynamic model with buffer cylinder when a failure occurs in the course of the lifting.

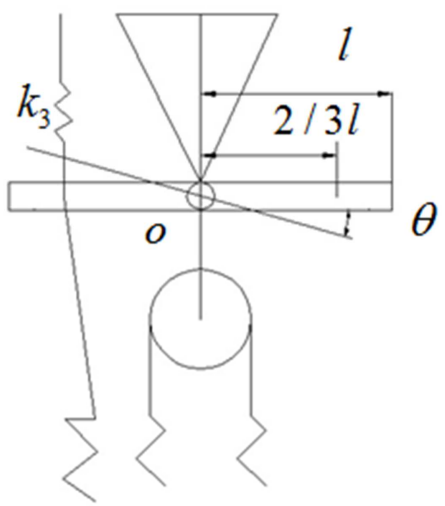

Fig. 5. The detailed drawing of the lifting mechanism with special structure at fault state.

The dynamic equation of the system is

$$
M \ddot{x}+C \dot{x}+K x=F
$$

Where,

$$
\begin{gathered}
x=\left[\begin{array}{lllll}
\theta_{1} & \theta_{2} & \theta & x_{1} & x_{2}
\end{array}\right]^{\mathrm{T}}, \\
F=\left[\begin{array}{lllll}
T_{t} & 2 T_{r} & 0 & 0 & 0
\end{array}\right]^{\mathrm{T}}, \\
M=\left[\begin{array}{ccccc}
J_{2} & 0 & 0 & 0 & 0 \\
0 & 2 J_{2} & 0 & 0 & 0 \\
0 & 0 & 0 & 0 & 0 \\
0 & 0 & 0 & m_{2} & 0 \\
0 & 0 & 0 & 0 & m_{2}
\end{array}\right], \\
K=\left[\begin{array}{ccccc}
k_{0} & -k_{0} & 0 & 0 & 0 \\
-k_{0} & k_{0}+\left(\frac{r}{i n}\right)^{2} & \frac{2}{3} \frac{l r k_{2}}{i n^{2}} & -\frac{r}{i n} k_{2} & -\frac{r}{i n} k_{2} \\
0 & \frac{r k_{2}}{i n} & \frac{2}{3} l\left(\frac{k_{2}}{n}+k_{3}\right) & -k_{2} & -k_{2} \\
0 & -\frac{r}{i n} k_{2} & \frac{2}{3} \frac{k_{2} l}{n} & k_{1}+k_{2} & k_{2} \\
0 & -\frac{r}{i n} k_{2} & \frac{2}{3} \frac{k_{2} l}{n} & k_{2} & k_{2}
\end{array}\right],
\end{gathered}
$$




$$
C=\left[\begin{array}{ccccc}
c_{0} & -c_{0} & 0 & 0 & 0 \\
-c_{0} & c_{0}+\left(\frac{r}{i n}\right)^{2} c_{2} & \frac{2}{3} \frac{l r c_{2}}{i n^{2}} & -\frac{r}{i n} c_{2} & -\frac{r}{i n} c_{2} \\
0 & \frac{r}{i n} c_{2} & \frac{2}{3} l\left(\frac{c_{2}}{n}+c_{3}\right) & -c_{2} & -c_{2} \\
0 & -\frac{r}{i n} c_{2} & \frac{2}{3} \frac{c_{2} l}{n} & c_{1}+c_{2} & c_{2} \\
0 & -\frac{r}{i n} c_{2} & \frac{2}{3} \frac{c_{2} l}{n} & c_{2} & c_{2}
\end{array}\right]
$$

\section{Analyzing Dynamic Characteristics}

As an integrated software package, SIMULINK is used to carry out dynamic system modeling and simulating. By using

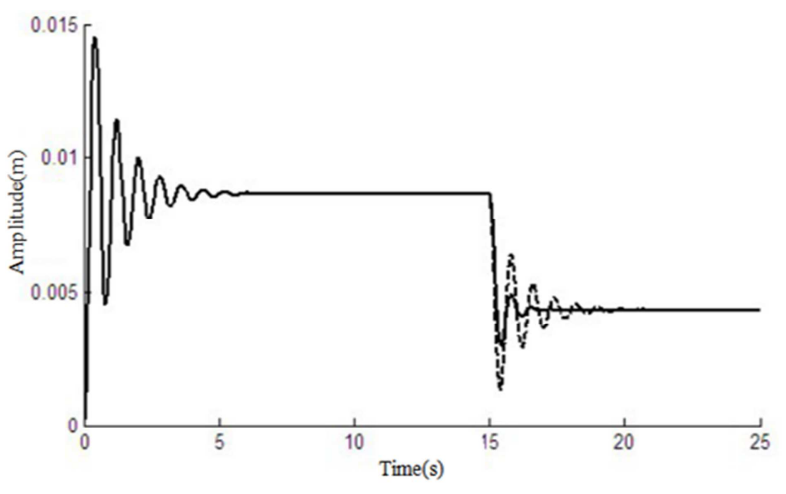

a. Time response of girder displacement.

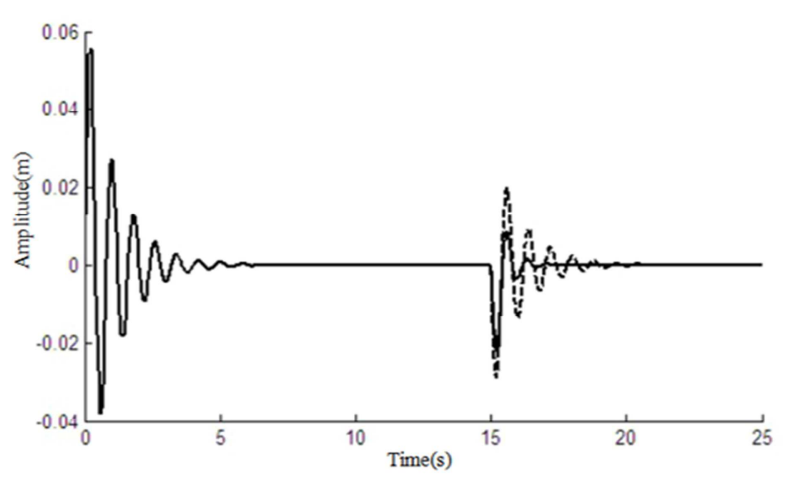

c. Time response of girder velocity.

Fig. 6. Time response of velocity and acceleration for the main girder and heavy loads with buffer cylinder and without buffer tank.

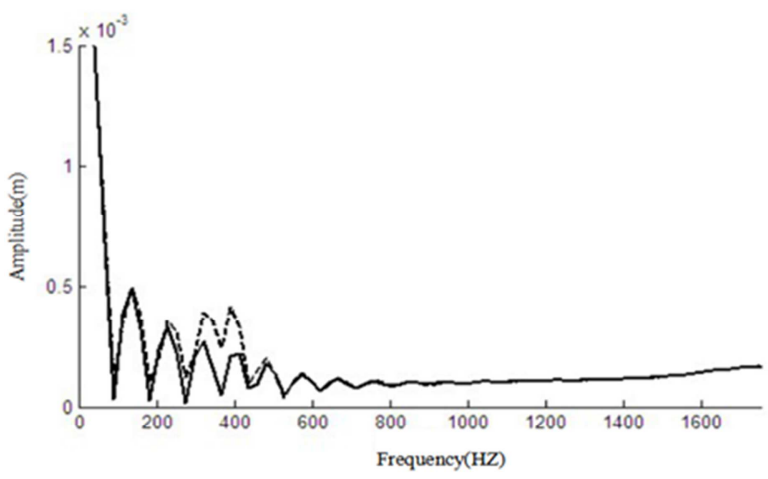

a. Frequency response of main girder displacement standard module of SIMULINK module library and setting parameters of corresponding module and its simulation system, and simulation system of lifting mechanism can be formed.

The method is simple and direct in modeling, calculation process and results analysis, and can fully guarantee the accuracy of result analysis, better solve coordination between accuracy of system modeling and numerical solution. At the same time, this method of the research can ascertain conveniently the dynamic load characteristics of hoisting mechanism of specific crane under different working conditions. It also overcomes the existing deficiency of methods for determining dynamic load coefficient, thus providing the reliable basis for the design of the crane.

\subsection{Simulation Parameters and Results}

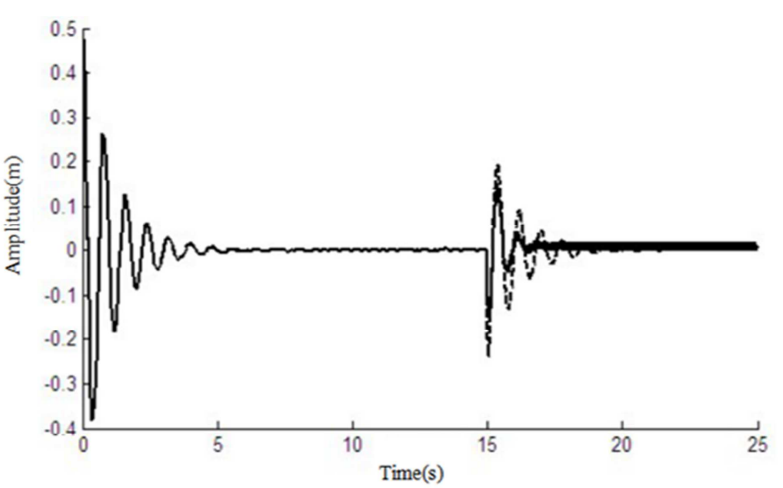

b. Time response of main girder acceleration.

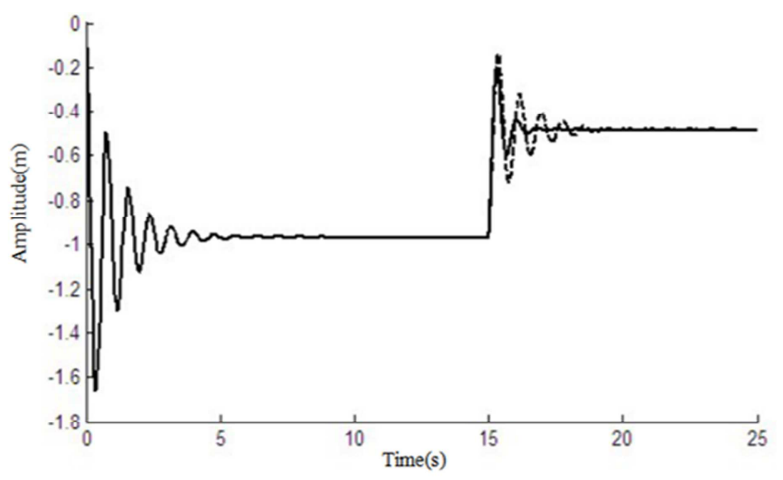

d. Time response of gravitational acceleration

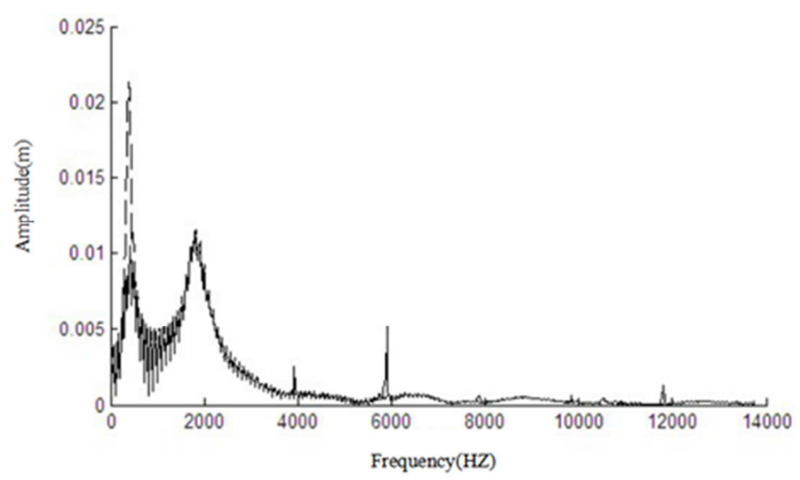

b. Frequency domain response of main girder acceleration 


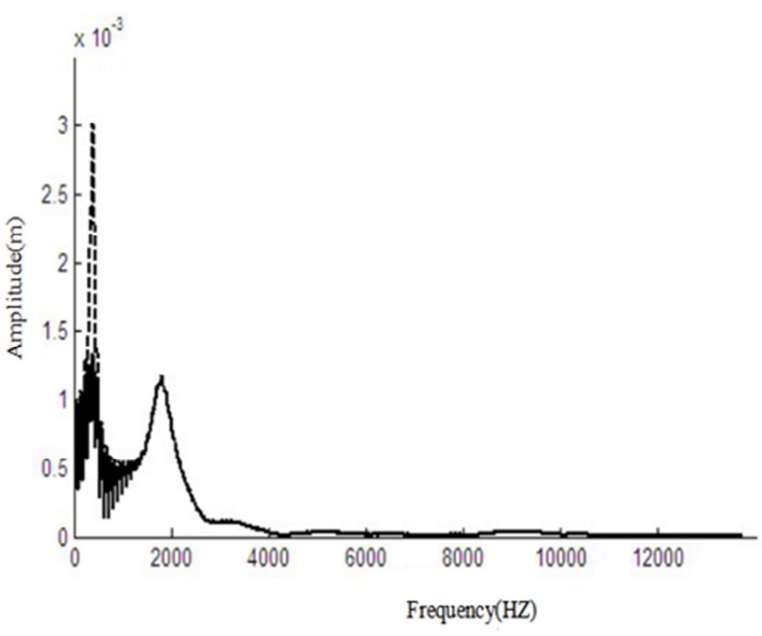

c. Frequency response of main girder velocity

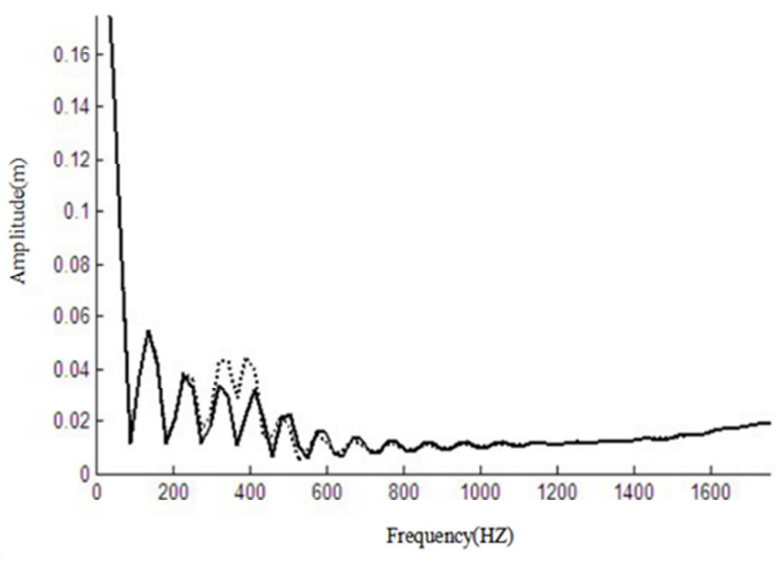

d. Frequency domain response of gravitational acceleration

Fig. 7. Frequency response of velocity and acceleration for the main girder and heavy loads with buffer cylinder and without buffer tank.

The simulation experiments aimed at the time domain and frequency response of the main beam and heavy loads with failure, when the cargo is lifted by the lifting mechanism for $15 \mathrm{~s}$. The system parameters has been properly processed in virtual processing, value is taken as $n=6, i=40, r=0.5$, $m_{1}=1.04 \times 10^{6} \mathrm{~kg}, m_{2}=1.29 \times 10^{5} \mathrm{~kg}, k_{1}=8.8 \times 10^{7} \mathrm{~N} / \mathrm{m}$, $k_{2}=2.76 \times 10^{6} \mathrm{~N} / \mathrm{m}, c_{1}=182110 \mathrm{~N} \cdot \mathrm{s} / \mathrm{m}, c_{2}=59000 \mathrm{~N} \cdot \mathrm{s} / \mathrm{m}$, $J_{1}=24.5 \mathrm{~kg} \cdot \mathrm{m}^{2} \quad, \quad J_{2}=0.7 \mathrm{~kg} \cdot \mathrm{m}^{2} \quad, \quad T_{t}=3933 \mathrm{~N} / \mathrm{m}$, $T_{r}=3071 \mathrm{~N} / \mathrm{m}$. Simulation results are shown in Fig. 6 and 7 and Table 1.

\subsection{Comparative Analysis of Simulation Results}

As seen from Fig. 6 and 7 and Table 1, compared with the system without the buffer cylinder device, when there are faults in the system, the amplitudes of the beam displacement, velocity, the acceleration and gravitational acceleration with the buffer cylinder device are reduced while the respective standard deviation are also reduced.

Table 1. Simulation results.

\begin{tabular}{|c|c|c|c|c|c|c|c|c|c|c|}
\hline \multirow{2}{*}{$\mathbf{a}$} & \multirow{2}{*}{ Evaluation index } & \multicolumn{3}{|c|}{ Displacement } & \multicolumn{3}{|c|}{ Velocity } & \multicolumn{3}{|c|}{ Acceleration } \\
\hline & & 1 & 2 & 3 & 1 & 2 & 3 & 1 & 2 & 3 \\
\hline \multirow{3}{*}{$\mathrm{b}$} & Max & 8.86 & 5.23 & $41 \%$ & 1.99 & 0.85 & $57.10 \%$ & 1.92 & 1.51 & $21.30 \%$ \\
\hline & Min & 1.37 & 1.78 & $30.30 \%$ & -2.88 & -2.21 & $23.30 \%$ & -2.39 & -1.53 & $35.90 \%$ \\
\hline & Standard deviation & 2.07 & 2.01 & $2.80 \%$ & 0.65 & 0.53 & $19.30 \%$ & 0.55 & 0.46 & $16.50 \%$ \\
\hline \multirow{3}{*}{$\mathrm{c}$} & $\operatorname{Max}$ & -1.4 & -2.14 & $53 \%$ & & & & & & \\
\hline & Min & -9.64 & -9.64 & 0 & & & & & & \\
\hline & Standard deviation & 2.31 & 2.26 & $2.25 \%$ & & & & & & \\
\hline
\end{tabular}

The marks in the table are 1. Without MRFD; 2. With MRFD; 3. Reduced Percentage

a. Structure, b. Main girder, c. Heavy load.

According to the simulation results, it is obvious that the dynamic load factor of wire ropes is decreased about $13.5 \%$, which reduced the maximum impact load of next rope within a very short time under the condition of accident, and at the same time, the dynamic load factor of the corresponding beam is also reduced accordingly. And the most obvious is that the time for fatigue happenning is greatly reduced, the vibration of the mechanism and the main girder structure is better controlled and relieved when the operation is a failure, as a consequence of which, the safety and stability of fault operation for nuclear power crane are improved and the accident rate and loss are reduced.

To sum up, special winding mode of drum system and setting buffer cylinder have an obvious effect on buffer shock of the crane that fails to work, which improves the safety and stability of the crane in fault operation.

\section{Conclusions}

In this paper, the dynamic models drawing of the normal operation of the lifting mechanism and in the case of failure were established, in view of the working characteristics, principle of the crane hoisting mechanism in nuclear power plant and the results of theoretical research. Based on the dynamic theory of the crane, ring crane of the lifting mechanism of ring crane in failure situation has been analyzed in detail, so as to establish the dynamic equation of the system. Dynamic computer simulation experiment has been conducted with SIMULINK tool in MATLAB, when a failure happens, dynamic response of lifting mechanism has been analyzed in detail, the moment that cargo was hanging.

1) Through dynamic theory the dynamic characteristics in 
this paper was studied, by simplifying crane lifting mechanism into many degrees of freedom elastic vibration system coupling the lifting mechanism and structure, in accordance with the actual situation of the project.

2) The corresponding model was established and the simulation experiment has been carried out, according to the special structure of crane hoisting mechanism, namely the buffer tank and wire rope winding system. Simulation results show that the system can effectively shorten the impact time of the load, effectually reduce the system's fatigue time and protect system.

3) By wire rope winding system and setting cushion cylinder of hoisting mechanism of nuclear power crane, safety and reliability of the system's fault operation are able to fully be guaranteed. It is widely used in various crane lifting mechanism, as a result of the advantages of this mechanism, it has the broad prospect for wide applications.

\section{Acknowledgements}

This research was supported by the Natural Science Foundation of Shanxi Province of China (Grant No. 2013011022-6), the National Science Foundation of China (Grant No. 51275329) and the National Science and Technology Major Project of China (Grant No. 2011 ZX 06001-015).

\section{References}

[1] P. N. Rizos, N. Aspragathos, A. D. Dimarogonas, Identification of crack location and magnitude in a cantilever beam from the vibration modes, Journal of Sound and Vibration 138 (3) (1990) 381-388.

[2] S. A. Neild, P. D. Mcfadden, M. S. Williams, A discrete model of a vibrating beam using a time-stepping approach, Journal of Sound and Vibration 239 (1) (2001) 99-121.

[3] I. Argatov, V. L. Popov, T. Rademacher, M. Zehn, A model of a breathing crack with relaxation damping, International Journal of Engineering Science 93 (2015) 46-50.

[4] T. G. Chondros, A. D. Dimarogonas, J. Yao, A continuous cracked beam vibration theory, Journal of Sound and Vibration 215 (1) (1998) 17-34.
[5] W. M. Ostachowicz, M. Krawczuk, Analysis of the effect of cracks on the natural frequencies of a cantilever beam, Journal of Sound and Vibration 150 (2) (1991) 191-201.

[6] V. Balamurugan, S. Narayanan, Shell finite element for smart piezoelectric composite plate/shell structures and its application to the study of active vibration control, Finite Elements in Analysis and Design 37 (9) (2001) 713-738.

[7] A. Zolfagharian, A. Noshadi, M. R. Khosravani, M. Z. Md. Zain, Unwanted noise and vibration control using finite element analysis and artificial intelligence, Appl Math Model 38 (9) (2014) 2435-2453.

[8] S. N. Mahmoodi, M. Ahmadian, Active vibration control with modified positive position feedback, J Dyn Syst Meas Control 131 (4) (2009) 1-8.

[9] M. I. Friswell, D. J. Inman, The relationship between positive position feedback and output feedback controllers, Smart Mater Struct 8 (3) (1999) 285.

[10] K. X. Li, J. Y. Gauthier, D. Guyomar, Structural vibration control by synchronized switch damping energy transfer, Journal of Sound and Vibration 330 (2011) 49-60.

[11] Q. Hu, G. Ma, Adaptive variable structure controller for spacecraft vibration reduction, IEEE Trans Aerosp Electron Syst 44 (3) (2008) 861-876.

[12] T. M. Seigler, J. B. Hoagg, Filtered dynamic inversion for vibration control of structures with uncertainty, J Dyn Syst Meas Control 135 (4) (2013).

[13] Y. H. Lin, M. W. Trethewey, Finite element analysis of elastic beams subjected to moving dynamic loads, Journal of Sound and Vibration 136 (2) (1990) 323-342.

[14] W. Xia, L. Wang, L. Yin, Nonlinear non-classical microscale beams: Static bending, postbuckling and free vibration, International Journal of Engineering Science 48 (2010) 2044-2053.

[15] H. Alli, A. Uar, Y. Demir, The solutions of vibration control problems using artificial neural networks, Journal of the Franklin Institute 340 (2003) 307-325.

[16] B. Saldivar, S. Mondie. Drilling vibration reduction via attractive ellipsoid method, Journal of the Franklin Institute 350 (2013) 485-502.

[17] Y. A. Huang, MATLAB 7.0/Simulink 6.0 Development of modeling and simulation of advanced engineering [M]. Beijing: Tsinghua University Press 12 (2005). 